\title{
Changes in Soil Microbial Community Structure and Functional Diversity in the Rhizosphere Surrounding Tea and Soybean
}

\author{
Xiaomin Qin, Chizhang Wei, Jinting Li, Yuanquan Chen, Hai Sheng Chen, Yi Zheng, \\ Yuqin Nong, Chunwen Liao, Xing Chen, Yanfei Luo, \\ Jinmei Lu, Zhiyun Zeng and Jinjian Wei
}

Received: $6^{\text {th }}$ July 2016 / Accepted: $9^{\text {th }}$ September 2016

\begin{abstract}
Field trial was conducted to evaluate the effects of different planting patterns (tea monocropping, tea and soybean intercropping, soybean monocropping) on microbial community structure and microbial functional diversity using Biolog technique. Results showed that intercropping treatment exhibited higher average well color development (AWCD), diversity indices and community functional diversity as compared with monocropping. The microbial utilization of 6 types of carbon source indicated some differences. Principal component analysis and cluster analysis demonstrated that intercropping treatment significantly changed the functional diversity of the soil microbial community, mainly depending on carbohydrates and carboxylic acids. Our findings suggested that soil microbial metabolic activities and functional diversity were significantly changed by tea-soybean intercropping.
\end{abstract}

Keywords: Intercropping, microbial functional diversity, rhizosphere, soybean, tea

\section{INTRODUCTION}

Tea (Camellia sinensis (L) O. Kuntze) is one of the most popular beverages in the world due to its health benefits that have been investigated especially in cancer prevention due to the presence of polyphenolic substances. (Saravanakumar et al., 2006; Yang et al., 2007; Khokhar and Magnusdottir, 2002), Mainly tea is classified into three main types namely, black tea, green tea and oolong tea according to its production process (Takeo, 1992). It is also a major cash crop in many developing countries, including China, India and Sri Lanka. Currently, China is the largest producer of tea, which accounts for $74.8 \%$ of global area and constitutes $41.6 \%$ of world production, and the total area under tea plantation is 2.74 million hectares (ITC, 2015).

Soil is an important substance for the growth of the tea plant as well as the absorption of nutrients and therefore, only rich soil can produce high quality tea (Lin et al., 2012). In the soil-plant ecosystem, the soil microorganisms are actively involved in the material and energy cycle of the ecosystem as one of the most active and decisive components (Doran and Zeiss, 2000), affected by a range of factors, such as planting patterns, soil type and climatic conditions. Planting patterns are one of the most important factors. Intercropping, as the essence of traditional agriculture, has been widely spread and applied in agricultural production. Compared to monocropping, numerous studies have shown that intercropping could increase efficiency of utilization of natural resources (Gao et al., 2009; He et al., 2013; Rivest et al., 2010), reduce disease, insect and weed (Hummel et al., 2009; Workayehu and Wortmann, 2011; AbdelMonaima and Abo-Elyousr, 2012), boost crop yield (Songa et al., 2007; Nataraj et al., 2010; 
Mao et al., 2012) and enhance soil microbial diversity (Hinsinger et al., 2011; Bainard et al., 2011). Therefore, the fact that how to achieve high quality and yield of tea and sustainable development by ecological regulation attract urgent attention.

As a traditional planting model, tea intercrops with different crops which could increase soil organic matter content and nutrition, improve the micro-climate of tea garden (Sun et al., 2011; Zhang et al., 2014), inhibit weeds and pests, maintain tea garden ecological balance (Kamunya et al., 2008; Ye et al., 2016), promote the tea growth and improve the tea quality and yield (Bore, 2005; Sedaghathoor and Janatpoor, 2012). Previous studies were concentrated primarily on the yield, quality, soil fertility and natural biological diversity, but changes in soil microbial community were not well understood in the tea intercropping system. Therefore, a field trial was conducted to explore whether intercropping improves the soil microbial community structure and function diversity, and provides a further scientific basis for the high yield and quality in the tea intercropping system.

\section{MATERIALS AND METHODS}

Study site: Field experiments were conducted in a tea garden (10 years old) of Guangxi South Subtropical Agricultural Science Research Institute at Longzhou, Chongzuo, Guangxi province in Southwest China $\left(22^{\circ} 21^{\prime} \mathrm{N}\right.$, $\left.106^{\circ} 46^{\prime} \mathrm{E}\right)$. This region has a typical subtropical monsoon climate with an average annual precipitation of $1273.6 \mathrm{~mm}$, an average annual temperature of $22^{\circ} \mathrm{C}$, an altitude of $125 \mathrm{~m}$ and an annual sunshine of $1251 \mathrm{~h}$. The soils are acid and red, and its initial properties were as follows: $\mathrm{pH}$ 5.86 \pm 0.21 ; soil organic matter $13.17 \pm 2.55$ $\mathrm{g} \mathrm{kg}^{-1}$; available nitrogen $128.44 \pm 2.64 \mathrm{mg} \mathrm{kg}^{-1}$; available phosphorus $30.68 \pm 2.43 \mathrm{mg} \mathrm{kg}^{-1}$; and available potassium $174.75 \pm 3.03 \mathrm{mg} \mathrm{kg}^{-1}$.

\section{Experiment design:}

Field trial was conducted with three planting treatments which consisted of tea monocropping, tea and soybean intercropping, soybean monocropping and three replications, on a total of 9 plots in a random block design, and the plot area was $24 \mathrm{~m}^{2}$. For intercropping, the planting ratios of tea and soybean were $2: 2$, i.e. every 2 rows tea intercropped with 2 rows soybean per plot. Tea were planted with a row width of $0.4 \mathrm{~m}$ and a plant spacing of $0.30 \mathrm{~m}$, and soybean with a row spacing of $0.3 \mathrm{~m}$ and a plant spacing of $0.15 \mathrm{~m}$. The fertilizers were applied according to the local custom, and nitrogen fertilizers rates of tea were applied 3 times a year, as at $40 \%$, $30 \%$ and $30 \%$, and that of soybean were applied two times at rates of $60 \%, 40 \%$. Phosphorus fertilizer $\left(\mathrm{P}_{2} \mathrm{O}_{5}\right)$, Potassium fertilizer $\left(\mathrm{K}_{2} \mathrm{O}\right)$ and organic resources were applied as base fertilizers. The fertilizer rates were same in each plot.

\section{Soil sampling}

The samplings took place at the beginning of July 2016. First, plant roots were taken off from the soil, shaken off the loose soil and then were brushed off for any remaining soil that was strongly adhered to the roots as rhizosphere soil. 4 plants per monocropping plot and 4 per intercropping plot were randomly selected and then the rhizosphere soils of 4 plants were mixed in one sample. Part of the soil was stored at $4{ }^{\circ} \mathrm{C}$ until being used for the microcosm experiment as described below, another part was air-dried, ground and passed through 1-mm and 2-mm mesh sieves for chemical analysis.

\section{Measurements}

Biolog Ecoplates (Biolog Inc., Hayward, CA, USA) were used to determine the soil microbial functional diversity based on the utilization of 31 carbon substrates (Garland and Mills, 1991). Fresh soils ( $10 \mathrm{~g})$ were extracted by shaking for $30 \mathrm{~min}$ at $200 \mathrm{rpm}$ with $90 \mathrm{ml} 0.85 \% \mathrm{NaCl}$. Tenfold dilutions were performed until the desired 
$\left(10^{-3}\right)$ dilution was reached. An aliquot (150 ul) of the diluted suspension was placed in each well of the Biolog Ecoplate using a multi-channel repetitive-dispensing pipette. The plates were incubated at $28^{\circ} \mathrm{C}$, and the absorbance at 590 $\mathrm{nm}$ was recorded at $24 \mathrm{~h}$ intervals for 10 days using the reader incorporated into the Biolog GEN III Micro Station TM (USA). Three replicates per treatment and sampling time were performed. The readings at $120 \mathrm{~h}$ were used for the statistical analysis.

The overall rate of substrate utilization by microorganisms was measured by calculating the Average Well Color Development (AWCD) for each plate;

$\mathrm{AWCD}=\sum\left(\mathrm{C}_{i}-\mathrm{R}_{i}\right) / 31$

where $\mathrm{C}_{i}$ is the $\mathrm{OD}$ in each carbon source well and $\mathrm{R}_{i}$ is the OD of control well. The microbial community diversity was calculated by the Shannon index;

$\mathrm{H}=-\sum \mathrm{P}_{i}\left(\ln \mathrm{P}_{i}\right)$

where $\mathrm{P}_{i}$ is calculated by subtracting the control from each substrate absorbance and then dividing this value by the total color change recorded for all 31 substrates, $\mathrm{P}_{i}=\left(\mathrm{C}_{i}-\mathrm{R}_{i}\right) / \sum$ $\left(\mathrm{C}_{i}-\mathrm{R}_{i}\right)$. The evenness was calculated as $\mathrm{E}=\mathrm{H} /$ $\ln$ [richness (S)], where the richness (S) referred to the number of substrates utilized.

\section{Statistical analysis}

Thecarbonsourcesutilization dataweresubjected to Principal Component Analysis (PCA) and Cluster Analysis (CA) using Microsoft Excel 2010 with Multibase. The microbial parameters for the different treatments were analyzed by Fisher's Least Significant Difference (LST) test at a significance level of 0.05 after verifying the significance by analysis of variance (ANOVA) using SPSS 19.0 software.

\section{RESULTS}

\section{Changes in average well color development (AWCD)}

The AWCD used to assess the utilization of overall carbon sources, is an important indicator that reflects the biological activity of soil microorganisms (Diosma et al., 2006). General shifts of the AWCD in different treated soils and changes within the incubation period were shown in Figure 01-A. The total utilization of different carbon sources by soil microorganisms appeared increasing tendency with cultural time, but that was different in different planting patterns. The changes of AWCD values were not obvious within 24h, and then increased rapidly until it tended to stabilize. During the whole culture period, the AWCD values were generally higher in intercropping treatment than that observed under monocropping treatment. Intercropping soybean exhibited the highest AWCD values, whilst the lowest AWCD values were found in monocropping tea.

As indicated in Figure 01-B, on the culture time of $120 \mathrm{~h}$, intercropping increased the AWCD values of tea and soybean by $11.52 \%$, $12.99 \%$ respectively when compared with monocropping, and with a significant difference between monocropping and intercropping soybeans. Moreover, the AWCD values of soybean were greater than that found in tea. The above analysis suggested that tea and soybean intercropping can promote the utilization of carbon sources by soil microorganisms to increase microbial metabolic activity. 

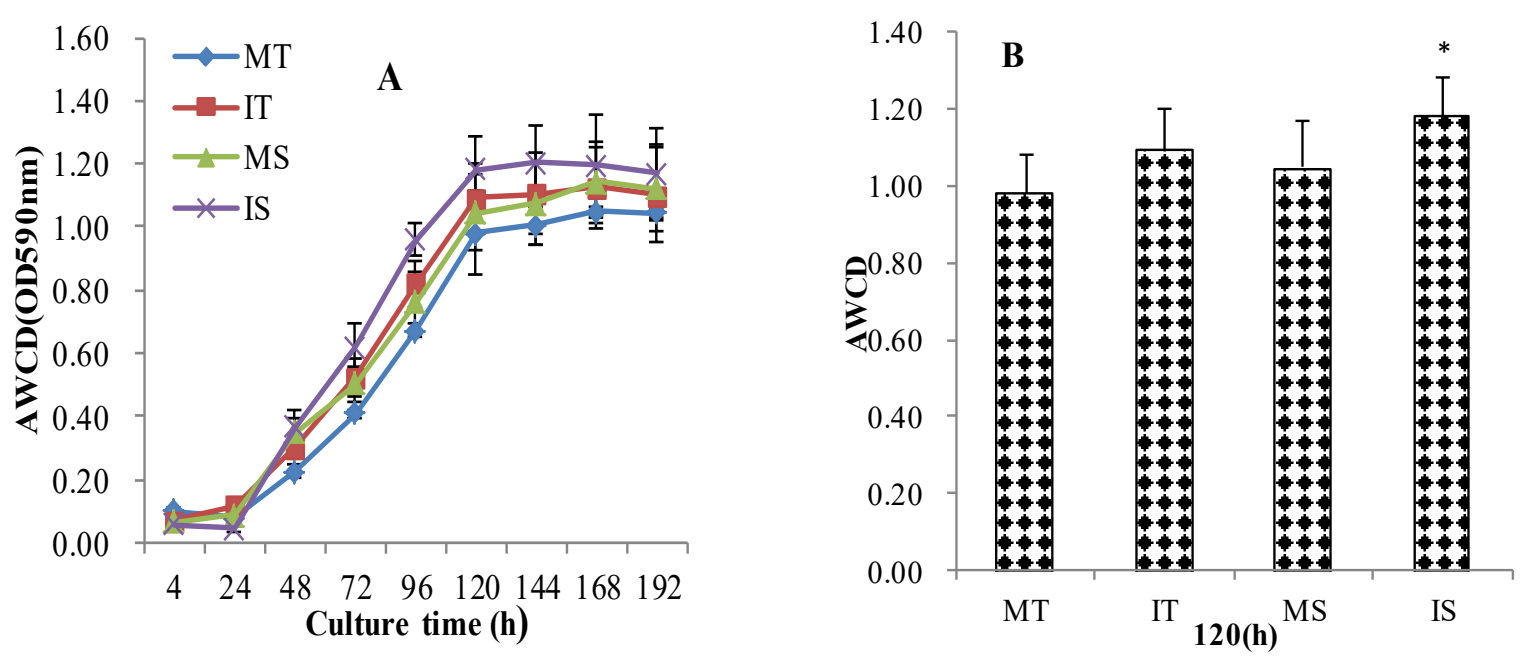

Figure 01: Effects of intercropping on average well color development (AWCD) of 31 carbon sources

Note: MT: Monocropping tea; IT: Intercropping tea; MS: Monocropping soybean; IS: Intercropping soybean. * mean significant difference between monocropping and intercropping pattern $(\mathrm{P}<0.05)$. The same below.

Differences in utilizing six types of carbon source by soil microorganisms

Soil microbial activity reflects the total changes of microbial community, and fails to show the detailed information about metabolism, so, study the differences in utilization of different carbon source contribute to more fully understanding the characteristics of microbial community metabolic function (Weber et al., 2007). As shown in Figure 02, the utilization of carbohydrates, carboxylic acids, amino acids and amides by soil microorganisms in the rhizosphere surrounding tea and soybean were stronger, the polymers and phenolic acids were weaker in monocropping and intercropping treatments.

As shown in Figure 03, the microbial utilization of 6 types of carbon source in the rhizosphere surrounding tea and soybean were affected by intercropping, but they had some differences. Compared with the monocropping, intercropping increased the microbial utilization of carboxylic acids, amides, polymers and phenolic acids in the rhizosphere surrounding tea by $45.14 \%, 72.92 \%, 24.47 \%, 141.67 \%$, and a significant difference existed in the utilization of polymers and phenolic acids. At the same time, the microbial utilization of carbohydrates, carboxylic acids, polymers and phenolic acids in the rhizosphere surrounding intercropping soybean were $1.02 \%, 53.19 \%$, $81.91 \%, 59.13 \%$ higher than that utilized in monocropping treatment, contributing to a significant difference found in the utilization of carboxylic acids, polymers and phenolic acids.

\section{Differences in utilizing single carbon source by soil microorganisms}

It can reflect soil microbial community structure bydetecting theutilization ofsoilmicroorganisms to single carbon source, which will be used to determine the microbial community functional diversity. As shown in Table 01, the utilization of soil microorganisms to $\alpha$-D-Lactose, $\beta$-Methyl-D-Glucoside, D-Xylose, i-Erythritol, D-Mannitol, L- $\alpha$-Glycerol Phosphate, L-Arginine, L-Phenylalanine, D-Galacturonic acid, D-Glucosaminic acid, $\alpha$-Ketobutyric acid, D-Malic acid, Tween 40, Cyclodextrin, Putrescine, 2-Hydroxy Benzoic acid and 4-Hydroxy Benzoic acid in intercropping tea were higher than that utilized in monocropping treatment. 


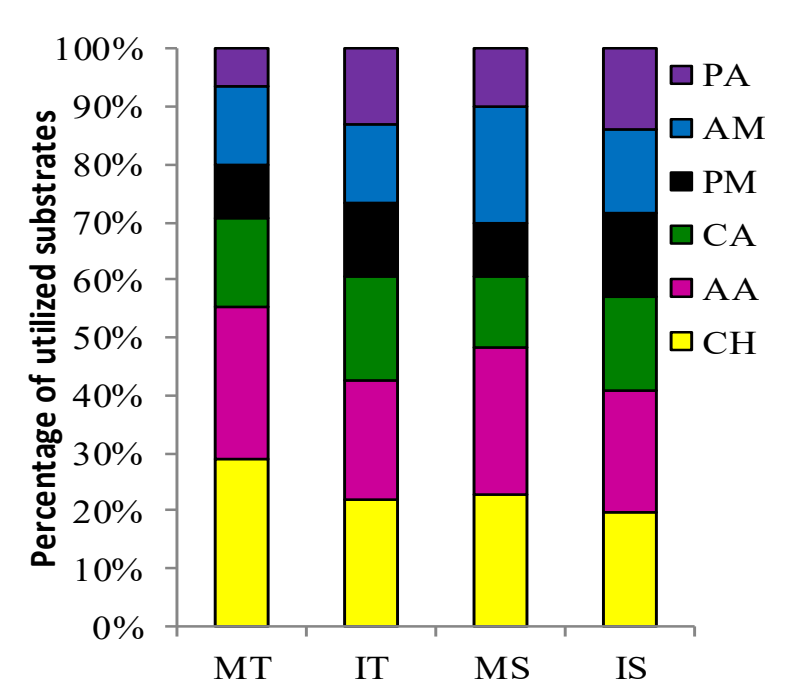

Figure 02: Percentage of utilized substrates by soil microorganisms

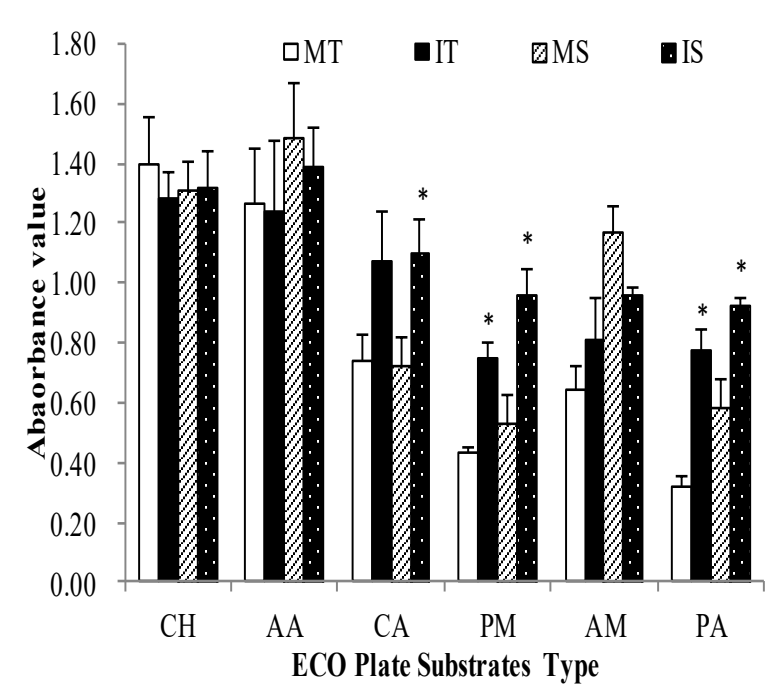

Figure 03: Utilization intensity of soil to six types of carbon source

Note: CH: carbohydrates; AA: amino acids; CA: carboxylic acids; PM: polymers; AM: amines/amides; PA: phenolic acids.

The microbial utilization of $\beta$-Methyl-DGlucoside, D-Xylose, i-Erythritol, D-Galactonic Acid $\gamma$-Lactone, L-Phenylalanine, L-Serine, Pyruvic Acid Methyl Ester, D-Glucosaminic acid, D-Galacturonic acid, $\gamma$-Hydroxybutyric acid, Itaconic acid, D-Malic acid, Tween
40, Cyclodextrin, Glycogen, Putrescine and 4-Hydroxy Benzoic acid in intercropping soybean were also stronger than that utilized in monocropping treatment. Furthermore, the microbial utilization of carbohydrates and carboxylic acids were more sensitive.

Table 01: Increased Carbon source type utilized by tea and soybean under intercropping

\begin{tabular}{cl}
\hline Crops & \multicolumn{1}{c}{ Carbon sources } \\
\hline & CH: $\alpha$-D-Lactose, $\beta$-Methyl-D-Glucoside, D-Xylose, i-Erythritol, D-Mannitol, L- $\alpha$-Glycerol \\
& Phosphate \\
AA: L-Arginine, L-Phenylalanine & CA: D-Galacturonic acid, D-Glucosaminic acid, $\alpha$-Ketobutyric acid, D-Malic acid \\
Tea & PM: Tween 40, Cyclodextrin \\
AM: Putrescine & PA: 2-Hydroxy Benzoic acid, 4-Hydroxy Benzoic acid \\
CH: $\beta$-Methyl-D-Glucoside, D-Xylose, i-Erythritol, D-Galactonic Acid $\gamma$-Lactone \\
AA: L-Phenylalanine, L-Serine \\
CA: Pyruvic Acid Methyl Ester, D-Glucosaminic acid, D-Galacturonic acid, $\gamma$-Hydroxybutyric \\
PM: Tween 40, Cyclodextrin, Glycogen \\
AM: Putrescine \\
PA: 4-Hydroxy Benzoic acid
\end{tabular}


Principal component analysis and cluster analysis of soil microbial diversity

\section{Principal component analysis}

To provide with a simpler interpretation of the utilization patterns for the 31 carbon resources of all treatments, we analyzed the utilization data with a partial least squares-discriminate enhance analysis (PLS-EDA). As shown in Figure $04 \mathrm{~A}$, there was a significant separation between monocropping and intercropping treatments on $\mathrm{PC} 1$, where the intercropping treatment was distributed mainly on the negative direction of $\mathrm{PC} 1$ and monocropping treatment was distributed mainly on the positive direction of PC1. However, no obvious difference was observed on $\mathrm{PC} 2$, which suggested that the carbon source utilization pattern of soil microbial community was significantly affected by intercropping. At the same time, the result indicated ten types of carbon source that were utilized strongly by soil microorganisms. The ten types of carbon source were namely, Tween 40, i-Erythritol, L- $\alpha$-Glycerol Phosphate, 2-Hydroxy Benzoic Acid, 4-Hydroxy Benzoic Acid, $\alpha$-Ketobutyric Acid, L-Arginine, L-Threonine, Phenyl ethylamine and Putrescine.

Initial load factors reflect the correlation coefficient between principal component and carbon source utilization; the higher the load factor, the greater effect of the carbon source

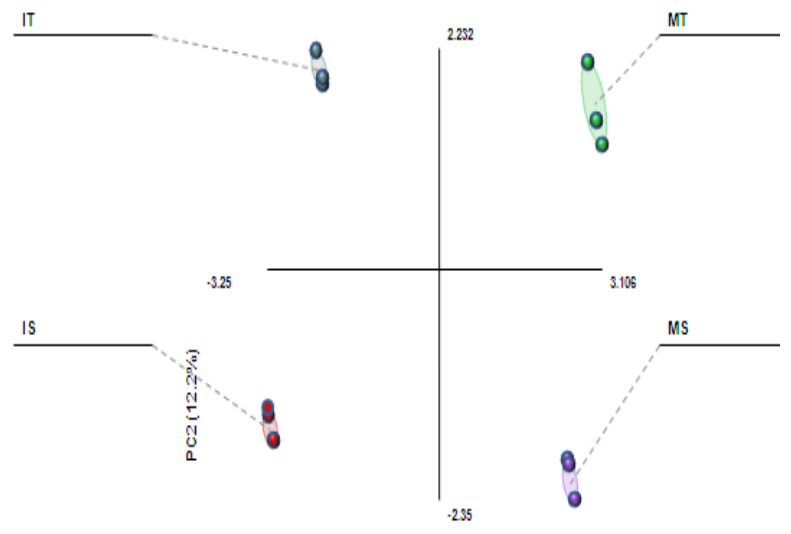

${ }^{\mathrm{PC}} \mathrm{A}^{26.856)}$ on the principal component. Choi et al., (1999) believes that load coefficients were greater than 0.18 or less than -0.18 on $\mathrm{PC} 1$ and $\mathrm{PC} 2$ could be considered to have a higher load. As shown in Table 02, there were 14 types of carbon source with the higher load on PC1, mainly including carbohydrates (4), amino acids (2), carboxylic acids (3), polymers (2), amines (2) and phenolic acids (1). While there were 9 types of carbon source with higher load on PC2, it mainly includes carbohydrates (3), amino acids (2), carboxylic acids (1), amines (1) and phenolic acids (2). The above analysis indicated that carbohydrates, carboxylic acids were the sensitive carbon sources that distinguish the differences between monocropping and intercropping treatments.

\section{Cluster analysis}

Cluster analysis is used to group the abstract objects into multiple categories with similarity that could be more intuitive to show the distance relationships among the objects. As shown in Figure 05, the average well color development (AWCD) of different treatments at $120 \mathrm{~h}$ was for cluster analysis. Result shows that intercropping and monocropping treatments is obviously divided into two categories that suggest soil microorganisms has different carbon source utilization pattern between monocropping and intercropping treatments.

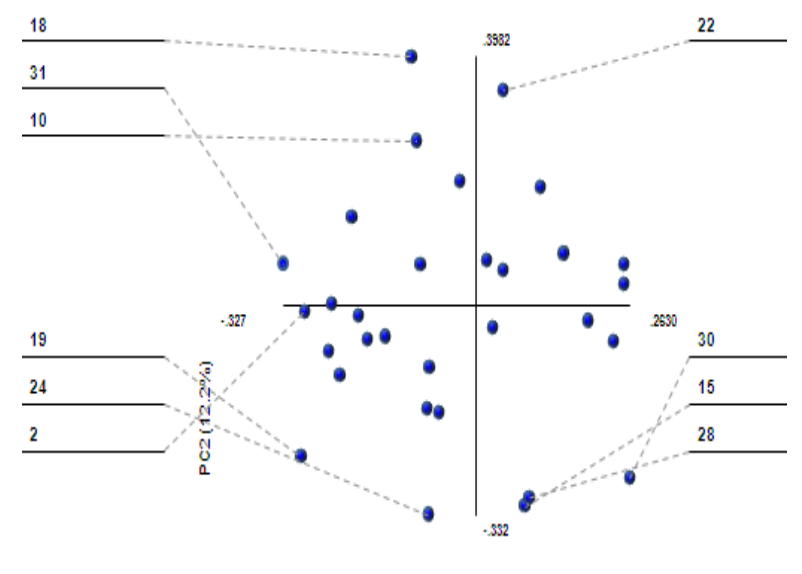

Be1 (26.8\%)

Figure 04: Principal Component analysis of carbon utilization profiles in soil microbial community of monocropping and intercropping treatments 
Table 02: Carbon substrates most heavily loaded on first two principal components (PC) in analysis of ECO micro-plate data

\begin{tabular}{cccc}
\hline Carbon sources & Substrates & PC1 & PC2 \\
\hline \multirow{4}{*}{ Carbohydrates } & D-Cellobiose & 0.25 & \\
& D-Xylose & -0.19 & \\
& N-Acetyl-D-Glucosamine & 0.19 & \\
& Glucose-1-Phosphate & 0.25 & 0.26 \\
& $\alpha-$ D-Lactose & & 0.19 \\
& D-Mannitol & & -0.32 \\
L- $\alpha$-Glycerol Phosphate & & \\
Amino acids & L-Asparagine & 0.23 & -0.33 \\
& L-Phenylalanine & -0.25 & -0.31 \\
& L-Arginine & & \\
L-Threonine & & \\
Carboxylic acids & D-Glucosaminic Acid & -0.20 & 0.34 \\
& D-Galacturonic Acid & -0.24 & \\
& D-Malic Acid & -0.21 & \\
Polymers & $\alpha-$ Ketobutyric Acid & & \\
& Tween 40 & -0.29 & -0.27 \\
Amines/amides & Cyclodextrin & -0.23 & -0.24 \\
& Phenyl ethylamine & 0.26 & 0.40 \\
\hline Phenolic acids & Putrescine & -0.33 & \\
& 4-Hydroxy Benzoic Acid & -0.30 & \\
\hline
\end{tabular}

The loading was $>0.18$ or $<-0.18$.

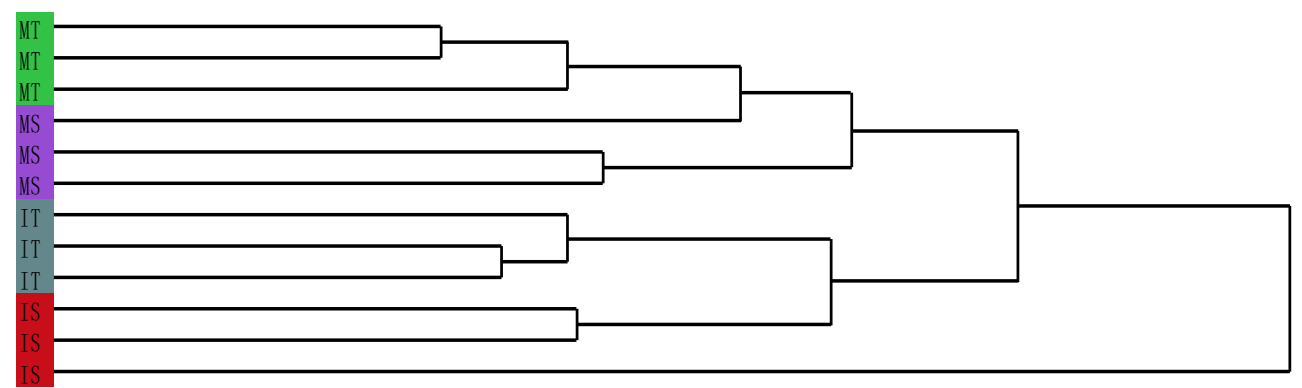

Figure 05: Cluster analysis of carbon utilization profiles

Microbial functional diversity index

The microbial diversity assessed by Shannon index (H), Simpson index (D), Evenness index (E) and Richness index (S) are given in Table 03. These soil microbial diversity indices in intercropping treatment were greater than that found in monocropping, and increased significantly soybeans soil microbial Shannon index $(\mathrm{H})$, Evenness index (E) and Richness index(S) by $7.91 \%, 4.85 \%$ and $10.14 \%$ as compared with monocropping. The increased diversity indicated that the carbon source utilization patterns were more diverse and the higher richness value indicated that a larger variety of substrates were utilized in soils under intercropping. 
Table 03: Effect of intercropping on soil microbial community diversity indices

\begin{tabular}{ccccc}
\hline \multirow{2}{*}{ Treatment } & $\begin{array}{c}\text { Shannon index } \\
(\mathrm{H})\end{array}$ & $\begin{array}{c}\text { Simpson index } \\
(\mathrm{D})\end{array}$ & $\begin{array}{c}\text { Evenness index } \\
(\mathrm{E})\end{array}$ & $\begin{array}{c}\text { Richness index } \\
(\mathrm{S})\end{array}$ \\
\hline MT & 3.041 & 0.944 & 0.898 & 29.67 \\
IT & 3.150 & 0.953 & 0.920 & 30.33 \\
MS & 2.971 & 0.945 & 0.908 & 26.33 \\
IS & $3.206^{*}$ & 0.956 & $0.952^{*}$ & $29.00^{*}$ \\
\hline
\end{tabular}

\section{DISCUSSION}

Plant diversity has a significant regulation on rhizosphere microbial diversity. Crops intercropping not only can improve the shoot ecological function, but also indirectly increases soil microbial diversity of the root (Song et al., 2007). Changes in AWCD values with time may be used to characterize mean microbial activity, the higher its value, the higher the metabolic activity (Konopka et al., 1998), and this effect was confirmed in our study. In our study, the microbial utilization of total carbon sources in the rhizosphere around tea and soybean met the growth rule of microbial cultivation, which appeared in the adaptation phase, logarithmic phase and stable phase. In addition, we also found that intercropping treatment increased the AWCD values of soil microorganisms in comparison with those obtained in monocropping soil and these were consistent with the results of previous studies where it was found that found that tea and white clover intercropping (Xu et al., 2008), wheat and faba bean intercropping (Dong et al., 2013; Yang et al., 2014), Soybean and Mulberry intercropping(Deng et al., 2015) could improve the total soil microbial metabolic activities and functional diversity.

Soil microbial Shannon index $(\mathrm{H})$, Simpson index (D), Evenness index (E) and Richness index (S) are commonly used to characterize the diversity of soil microbial, and can reveal the differences of soil microbial species and function (Tian et al., 2003). Nai et al., (2013) where it was found that intercropping significantly improves rhizosphere soil microbial Shannon diversity index and richness index around faba bean and wheat. When compared with monocropping. Kihara et al., (2012) it also reported that microbial Shannon index $(\mathrm{H})$, Simpson index (D) and Richness index (S) were higher in maize-soybean intercropping systems than that observed under monocropping. In our study, the soil microbial Shannon index (H), Simpson index (D), Evenness index (E) and Richness index (S) were higher in tea-soybean intercropping systems than that obtained under monocropping, and the increases of soil microbial diversity indices around soybean were more obvious and these were consistent with the results of previous studies. Lu and Zhang (2006) had reported that the root exudates and plant residues could provide a favorable environment as well as carbon sources and energy for the growth of soil microorganisms. So, the intercropping systems could improve the microbial community metabolic activity and functional diversity may be due to different crops root interaction which will release more abundant root exudates in intercropping system where it could provide more nutrients for growth and reproduction of soil microorganisms that promote the formation of soil microbial community structure diversity than monocropping.

In our study, the microbial utilization of carboxylic acids, amides, polymers and phenolic acids in the rhizosphere surrounding tea in intercropping treatment were greater than monocropping and the utilization of soil microorganisms to carbohydrates, carboxylic acids, polymers and phenolic acids in the rhizosphere surrounding soybean in intercropping were higher than monocropping. 
Furthermore, there were 17 types of single carbon source by soil microorganisms in the rhizosphere surrounding tea and soybean in intercropping treatment were stronger than monocropping treatment respectively. Others were weaker than monocropping. It showed that utilization of these carbon sources strengthen or weaken in relation to the amounts of soil microbial population that could utilized such carbon sources to be increased or decreased and these due to the changes and accumulation in crops root exudates under intercropping may enhance the soil microbial functional diversity and may simultaneously lead to some feedback regulation.

Principal component analysis explained the differences in microbial utilization of carbon sources in different treatments. The composition of carbohydrates, amino acids and phenolic acids in root exudates was changed in rice-watermelon systems (Hao et al., 2010). Carbohydrates, carboxylic acids and polymers are the main carbon sources for microbial utilization in the mulberry-soybean intercropping system that could be used as the basis for distinguishing the microbial utilization of carbon sources in different planting patterns (Li et al., 2012). In our study, principal component analysis result suggested that a significant difference was found in monocropping and intercropping treatments on PC1, but no obvious difference on PC2. These indicated that tea-soybean intercropping changed the soil microbial community functional diversity. This was caused by the differences in microbial utilization of the carbon sources with higher load on the $\mathrm{PCl}$, and the main carbon sources with higher load on PC1were the carbohydrates and carboxylic acids. These reflected the carbohydrates and carboxylic acids were the sensitive carbon sources to distinguish difference between monocropping and intercropping treatments that showed changes in soil microbial functional diversity in tea-soybean intercropping system. These were caused by the differences in microbial utilization of carbohydrates and carboxylic acids that suggested that the tea and soybean intercropping may change the composition of carbohydrates and carboxylic acids.

Cluster analysis results also indicated that the soil microorganisms in monocropping and intercropping treatments were divided into two categories, and a significant difference was found in them that suggested soil microorganisms had a different carbon source utilization pattern.

\section{CONCLUSIONS}

Tea and soybean intercropping is an important practice of multiple cropping and stereo cultivation for improving tea quality and yield in China. The effects of such an intercropping system on the soil microbial properties remain unclear. In our study, the soil microbial metabolic activities (AWCD) and diversity indices in the rhizosphere surrounding tea and soybean in intercropping were increased compared with those found under monocropping. Principal component analysis and Cluster analysis results suggested that intercropping significantly changed soil microbial community metabolic activities and functional diversity depending mainly on carbohydrates and carboxylic acids. However, the microbial utilization of 6 types of carbon source had some differences. Therefore, future researches should be focused on efforts to gain in-depth knowledge about the tea-soybean intercropping system underlying the changes in soil microbial properties and root exudates, particularly the interrelation between diversity properties and tea quality.

\section{ACKNOWLEDGEMENTS}

We thank the Public Basic Research Program of Guangxi (GXNYRKS) (201608, 201501, and 201609) for the financial support. 


\section{REFERENCES}

Abdel-Monaima, M.F. and Abo-Elyousr K.A.M. (2012). Effect of preceding and intercropping crops on suppression of lentil damping-off and root rot disease in New Valley-Egypt. Crop Protection 32: 41-46. https:/doi.org/10.1016/j.cropro.2011.10.011

Bainard, D., Klironomos, J. and Gordon, A. (2011). Arbuscular mycorrhizal fungi in tree-based intercropping systems: A review of their abundance and diversity. Pedobiologia 54: 57-61. https:/doi.org/10.1016/j.pedobi.2010.11.001

Bore, J.K. (2005). Effects of intercrops on yields of young tea. Tea 26: 52-54.

Choi, K.H. and Dobbs, F.C. (1999). Comparison of two kinds of Biolog micro plates (GN and ECO) in their ability to distinguish among aquatic microbial communities. Journal of Microbiological Methods 36: 203-213. https:/doi.org/10.1016/S0167-7012(99)00034-2

Doran, J.W. and Zeiss, M.R. (2000). Soil health and sustainability: managing the biotic component of soil quality. Applied Soil Ecology 15: 3-11. https:/doi.org/10.1016/S0929-1393(00)000676

Diosma, G., Aulicino, M., Chidichimo, H. and Balatti P.A. (2006). Effect of tillage and N fertilization on microbial physiological profile of soils cultivated with wheat. Soil \&Tillage Research 91: 236-243. https:/doi.org/10.1016/j.still.2005.12.008

Dong, Y., Dong, K., Tang, L., Zheng, Y., Yang, Z.X., Xiao, J.X., Zhao, P. and Hu, G.B. (2013). Relationship between rhizosphere microbial community functional diversity and faba bean Fusarium wilt occurrence in wheat and faba bean intercropping system. Acta Ecologica Sinica 33: 7445-7454. https:/doi.org/10.5846/stxb201208281214

Deng, W., Hu, X.M., Yu, C., Ye, C.H., Li, Y., Xiong, C. and Du, H. (2015). Impact of Intercropping Soybean in Mulberry Field on Soil Microbial Diversity. Acta Sericologica Sinica 41: 9971003.

Gao, Y., Duan, A., Sun, J., Li, F., Liu, Z., Liu, H. and Liu, Z. (2009). Crop coefficient and wateruse efficiency of winter wheat/spring maize strip intercropping. Field Crops Research 111: 65-73. https:/doi.org/10.1016/j.fcr.2008.10.007

Garland, J.L. and Mills, A.L. (1991). Classification and characterization of heterotrophic microbial communities on the basis of patterns of community-level sole carbon source utilization. Applied and Environmental Microbiology 57: 2351-2359.

He, Y., Ding, N., Shi, J.C., Wu, M., Liao, H. and Xu, J.M. (2013). Profiling of microbial PLFAs: Implication for interspecific interactions due to intercropping which increase phosphorus uptake in phosphorus limited acidic soils. Soil Biol Biochem 57: 625-634. https:/doi. org/10.1016/j.soilbio.2012.07.027

Hinsinger, P., Betencourt, E., Bernard, L., Brauman, A., Plassard, C. and Shen, J.B. (2011). P for two, sharing a scarce resource: Soil phosphorus acquisition in the rhizosphere of intercropped species. Plant Physiology 156: 1078-1086. https:/doi.org/10.1104/pp.111.175331 
Hao, W.Y., Ren, L.X., Ran, W. and Shen, Q.R. (2010). Allelopathic effects of root exudates from watermelon and rice plants on Fusarium oxysporum f.sp. niveum. Plant Soil 336: 485-497. https:/doi.org/10.1007/s11104-010-0505-0

Hummel, J.D., Dosdall, L.M., Clayton, G.W., Turkington, T.K., Lupwayi, N.Z., Harker, K.N. and Donovan, J.T. (2009). Canola-wheat intercrops for improved agronomic performance and integrated pest management. Agronomy Journal 101: 1190-1197. https:/doi.org/10.2134/ agronj2009.0032

International Tea Committee (ITC). 2015. Annual Bulletin of Statistics.

Khokhar, S. and Magnusdottir, S.G.M. (2002). Total phenol, catechin and caffeine contents of tea commonly consumed in the United Kingdom. J. Agric. Food Chem. 50: 565-570. https:/doi. org/10.1021/jf0101531

Kamunya, S.M., Wachira, F.M., Lang'at, J. and Sudoi, V. (2008). Integrated management of root knot nematode (Meloidogyne spp.) in tea (Camellia sinensis) in Kenya. International Journal of Pest Management 54: 129-136. https:/doi.org/10.1080/09670870701757896

Konopka, A., Oliver, L. and Turco, R.F. (1998). The use of carbon substrate utilization patterns in environmental and ecological microbiology. Microbial Ecology 35: 103-115. https:/doi. org/10.1007/s002489900065

Kihara, J., Martius, C., Bationo, A., Thuita, M., Lesueur, D., Herrmann, L., Amelung, W. and Vlek, P.L.G. (2012). Soil aggregation and total diversity of bacteria and fungi in various tillage systems of sub-humid and semi-arid Kenya. Applied Soil Ecology 58: 12-20. https:/doi. org/10.1016/j.apsoil.2012.03.004

Lin, S., Zhuang, J.Q., Chen, T., Zhamg, A.J., Zhou, M.M. and Lin, W.X. (2012). Analysis of nutrient and microbial Biolog function diversity in tea soils with different planting years in Fujian Anxi. Chinese Journal of Eco-Agriculture 20: 1471-1477. https:/doi.org/10.3724/ SP.J.1011.2012.01471

Lu, Y.H. and Zhang F. S. (2006). The advances in rhizosphere microbiology. Soil 38: 113-121.

Li, X., Zhang, H.H., Yue, B.B., Jin, W.W., Xu, N., Zhu, W.X. and Sun, G.Y. (2012). Effects of mulberry-soybean intercropping on carbon-metabolic microbial diversity in saline-alkaline soil. Chinese Journal of Applied Ecology 23: 1825-1831.

Mao, L.L., Zhang, L.Z., Li, W.Q., Werf, W.V.D., Sun, J.H., Spiertz, H. and Li, L. (2012). Yield advantage and water saving in maize/pea intercrop. Field Crops Research 138: 11-20. https:/ doi.org/10.1016/j.fcr.2012.09.019

Nataraj, D., Shashidhar, K.S., Vinoda, K.S., Chandrashekara, C. and Kalyanamurthy. (2010). Profitability and potentiality of baby corn based leguminous vegetable intercropping system. Environment and Ecology 28: 1433-1436.

Nai, F.J., Wu, L.H., Liu, H.Y., Ren, J., Liu, W.X. and Luo, Y.M. (2013). Effects of intercropping Sedum plumbizincicola and Apium graceolens on the soil chemical and microbiological properties under the contamination of zinc and cadmium from sewage sludge application. Chinese Joumal of Applied Ecology 24: 1428-1434. 
Rivest, D., Cogliastro, A., Bradley, R. and Olivier, A. (2010). Intercropping hybrid poplar with soybean increases soil microbial biomass, mineral N supply and tree growth. Agroforestry Systems 80: 33-40. https:/doi.org/10.1007/s10457-010-9342-7

Saravanakumara, D., Vijayakumarc, C., Kumarb, N. and Samiyappana, R. (2006). PGPR-induced defense responses in the tea plant against blister blight disease. Crop Protection 26: 556565. https:/doi.org/10.1016/j.cropro.2006.05.007

Sedaghathoor, S. and Janatpoor, G. (2012). Study on effect of soybean and tea intercropping on yield and yield components of soybean and tea. Journal of Agricultural and Biological Science 7: 664-671.

Songa, J.M., Jiang, N., Schulthess, F. and Omwega, C. (2007). The role of intercropping different cereal species in controlling lepidopteran stemborers on maize in Kenya. Journal of Applied Entomology 131: 40-49. https:/doi.org/10.1111/j.1439-0418.2006.01116.x

Sun, Y.N., Liang, M.Z., Xia, L.F., Wang, L., Cai, L., Yang, S.M. and Chen, M. (2011). Effects of intercropping different crops in tea garden on soil nutrients. Southwest China Journal of Ggriculture Sciences 24: 149-153.

Song, Y.N., Zhang, F.S., Marschner, P., Fan, F.L., Gao, H.M., Bao, X.G., Sun, J.H. and Li, L. (2007). Effect of intercropping on crop yield and chemical and microbiological properties in rhizosphere of wheat (Triticum aestivum L.), maize (Zea mays L.), and faba bean (Vicia faba L.). Biology \& Fertility of Soils 43: 565-574. https:/doi.org/10.1007/s00374-006-0139-9

Takeo, T. (1992). Green and semi-fermented teas. In: Willson, K.C., Clifford, M.N. (Eds.), Tea: Cultivation to Consumption. Chapman and Hall, London, pp. 413-510. https:/doi. org/10.1007/978-94-011-2326-6_13

Tian, C.J., Chen, J.K. and Zhong, Y. (2003). Phylogentic diversity of microbes and its perspectives in conservation biology. Chinese Journal of Applied Ecology 14: 609-612.

Weber, K.P., Grove, J.A., Gehder, M., Anderson, W.A. and Legge, R.L. (2007). Data transformations in the analysis of community-level substrate utilization data from micro plates. Journal of Microbiological Methods 69: 461-469. https:/doi.org/10.1016/j.mimet.2007.02.013

Workayehu, T. and Wortmann, C.S. (2011). Corn-bean intercrop weed suppression and profitability in Southern Ethiopia. Agronomy Journal 103: 1058-1063.https:/doi.org/10.2134/ agronj2010.0493

Xu, H.Q., Xiao, R.L., Song, T.Q., Luo, W., Ren, Q. and Huang, Y. (2008). Effects of mulching and intercropping on the functional diversity of soil microbial communities in tea plantations. Biodiversity Science 16: 166-174. https:/doi.org/10.3724/SP.J.1003.2008.07093

Yang, C.S., Lee, M.J., Chen, L. and Yang, G.Y. (1997). Polyphenols as inhibitors of carcinogenesis. Environ. Health Perspect. 105: 4971-4976.https:/doi.org/10.1093/carcin/18.12.2361

Ye, H.X., Han, S.J. and Han, B.Y. (2016). The abundance of pests and natural enemies in the tea plantation intercropped with citrus, waxberry, and snake gourd fruit plants. Journal of Anhui Agricultural University 43: 1-4. 
Yang, Z.X., Tang, L., Zheng, Y., Dong, Y. and Dong, K. (2014). Effects of different wheat cultivars intercropped with faba bean on faba bean Fusarium wilt, root exudates and rhizosphere microbial community functional diversity. Journal of Plant Nutrition and Fertilizer 20: 570 579 .

Zhang, X.Q., Chen, J. and Liang, Y.F. (2014). Advances in the effects of intercropping on ecological factors, growth and economic benefits of young tea garden. Guizhou Agriculture sciences 42: 67-71. 